\title{
Bacteriemia por Leuconostoc spp: Descripción de un caso
}

\author{
A case of bacteremia caused by Leuconostoc spp
}

\begin{abstract}
Virginia González Hidalgo ${ }^{1}$, María Ángeles Sánchez Pérez², María Teresa Nieto Riesco², María Paz Varillas López²
${ }^{1}$ Unidad de Medicina Interna del Hospital Virgen del Puerto. Servicio Extremeño de Salud. Plasencia (Cáceres). España. ${ }^{2}$ Unidad de Cuidados Paliativos del Hospital Virgen del Puerto. Servicio Extremeño de Salud. Plasencia (Cáceres). España.
\end{abstract}

\section{RESUMEN}

Leuconostoc spp es una bacteria tipo coco gram-positivo; recientemente se ha demostrado su potencial patógeno, sobre todo en pacientes inmunodeprimidos y con factores de riesgo como la patología oncológica. Dentro de los cuadros clínicos que pueden producir destacan: neumonía, meningitis, endocarditis o bacteriemia, siendo estas últimas las más frecuentes. Cabe destacar su resistencia intrínseca al tratamiento con vancomicina, siendo el tratamiento de elección en estos casos la penicilina y otros fármacos pertenecientes al grupo de los beta-lactámicos.

Palabras clave: Leuconostoc, Vancomicina, inmunodeprimido, tumor, bacteriemia.

\section{INTRODUCCIÓN}

Las bacterias pertenecientes al grupo Leuconostoc son cocobacilos gram-positivos no esporulados incluidos en la familia Streptococcaceae. Se tratan de organismos que dentro del ámbito clínico no se han considerado especies patógenas hasta hace aproximadamente dos décadas, momento en que fueron aisladas como organismos oportunistas en algunos pacientes inmunocomprometidos, patologías gastrointestinales y portadores de dispositivos intravenosos, en los que producían, entre otros cuadros clínicos, bacteriemia, sepsis, neumonía, meningitis o endocarditis ${ }^{1,2}$.

\section{DESCRIPCIÓN DEL CASO}

Se presenta el caso de un varón de 59 años, alérgico a beta-lactámicos, exfumador de 2 paquetes/día, hipertenso y con antecedentes de adenocarcinoma de recto estadio IV, intervenido quirúrgicamente mediante resección anterior ultra-baja con anastomosis colo-anal y linfadenectomía, en tratamiento con quimioterapia paliativa (último ciclo tres meses antes). Historia de dolor abdominal crónico que precisó de múltiples ingresos hospitalarios en relación con cuadros de pseudo-obstrucción intestinal.

Es hospitalizado por cuadro de fiebre de hasta $40^{\circ} \mathrm{C}$, dolor abdominal, tos y expectoración de color oscura. En la exploración física presentaba palidez cutánea y abdomen doloroso a la palpación generalizada, sin defensa abdominal. Resto de exploración física dentro de la normalidad. Analíticamente destacaba leucocitosis con neutrofilia [19.200 x10E9/L (N $68.9 \%)]$, proteína C reactiva de $254 \mathrm{mg} / \mathrm{dL}$ y procalcitonina de $1.27 \mathrm{ng} / \mathrm{ml}$. La radiografía de tórax mostraba una tenue condensación a nivel basal izquierdo (Figura 1). Durante el episodio febril se procedió a la extracción de dos muestras de hemocultivos, en los que se aislaron Leuconostoc spp, estableciéndose antibioterapia con clindamicina, dadas las alergias medicamentosas del paciente.

\begin{abstract}
Leuconostoc spp is a gram-positive bacterium which pathogenic potential has been recently demonstrated, especially in immunocompromised patients and in those with risk factors la oncologic diseases. It can cause infections presented as pneumonia, meningitis, endocarditis or bacteremia, being the last two the most frequent ones. It can be highlighted its intrinsic resistance to vancomycin, which makes penicillin and other beta-lactam antibiotics the first treatment options.
\end{abstract}

Keywords: Leuconostoc, Vancomycin, immunocompromised, tumor, bacteremia.

La evolución clínica, radiológica y analítica del paciente tras tratamiento antibiótico fue favorable, con desaparición de la fiebre, por lo que se procedió al alta con diagnóstico de sepsis por Leuconostoc, junto con infección respiratoria y dolor abdominal asociado a cuadros sub-oclusivos.

\section{DISCUSIÓN}

Leuconostoc spp es un coco gram-positivo que no ha sido considerado clínicamente como patógeno hasta que en 1985 fue descrito por Buu-Hoy et al el primer caso de infección en humanos producida por dicho microorganismo ${ }^{3}$. Principalmente se atribuyó la predisposición a esta infección factores que favorecían la inmunosupresión y a la presencia de patología tumoral maligna ${ }^{2,4}$. Estudios posteriores, como el de Bou $\mathrm{G}$ et al ${ }^{5}$ analizaron más a fondo los factores de riesgo asociados a dicho patógeno, resultando como principal factor desencadenante el ser portador de nutrición parenteral; siendo otros predisponentes la presencia de alteraciones que producen una pérdida de integridad a nivel de la mucosa del tracto gastrointestinal o de la barrera mucocutánea, por lo que, aunque es una infección frecuentemente asociada a inmunodepresión, puede ocasionar patología en pacientes inmunocompetentes ${ }^{6,7}$.

En nuestro caso, la bacteriemia por Leuconostoc se produjo en un paciente con antecedentes de adenocarcinoma de recto intervenido quirúrgicamente, factor predisponente descrito en la literatura ${ }^{8}$, en un estudio realizado a sujetos con infección por dicho microorganismo, en el que se observaron casos de pacientes con tumores a nivel colo-rectal y rectal, los cuales, adicionalmente, presentaban antecedentes de cuadros de pseudo-obstrucción intestinal, como en este caso (con múltiples ingresos por lo mismo) ${ }^{7,8}$.

No obstante, dado los múltiples factores de riesgo que nuestro paciente presentaba: adenocarcinoma de recto, la pérdida de integridad y extensión intestinal, así como haber re- 
Fig.1. Radiografía de tórax en proyecciones anteroposterior (A) y lateral (B) de nuestro paciente, donde podemos observar una condensación a nivel basal izquierdo, correspondiente a una neumonía producida por Leuconostoc spp.

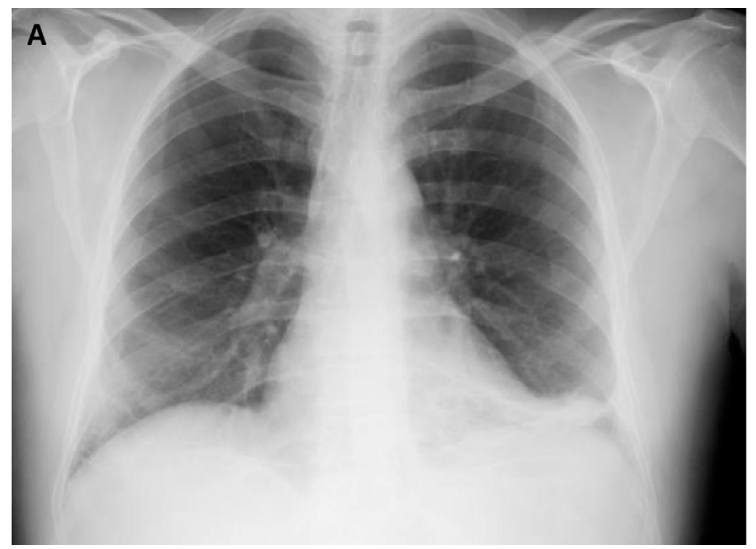

cibido tratamiento quimioterápico dentro de los tres meses previos al desarrollo de la bacteriemia, factor descrito por Lee $M$ et af, no se puede afirmar la existencia de un factor predisponente definido.

Dentro del género Leuconostoc, existen múltiples especies como L. citreum, L. lactis, L. garlicum o L. mesenteroides, entre otras, siendo esta última la más frecuentemente descrita. Los cuadros clínicos que pueden llegar a producir incluyen bacteriemia, sepsis, meningitis, neumonía, endocarditis u osteomielitis. La bacteriemia y la sepsis son las patologías más habituales en la práctica clínica, siendo la fiebre en pacientes con condiciones predisponentes la manifestación inicial más frecuente ${ }^{2,8,9,10,11}$, hecho que podemos ejemplificar con nuestro caso, en el que el sujeto presentó una clínica inicial consistente en fiebre alta y posterior desarrollo de infección respiratoria con repercusión clínica generalizada y diseminación hematológica bacteriana.

Una de las peculiaridades de este microorganismo radica en la resistencia intrínseca que presenta al tratamiento con vancomicina, a pesar de tratarse de un coco gram-positivo. Dicha característica se explica porque el pentapéptido de la pared celular, lugar de unión de la vancomicina, finaliza en alanina-lactato en lugar de alanina-alanina. Dicha resistencia parece estar mediada de modo cromosómico ${ }^{2,8}$. Además, esta característica parece que tiene un papel aún sin dilucidar, en la predisposición de infección por dicha bacteria, en casos con antecedentes de tratamiento con dicho antibiótico ${ }^{9}$. Por tanto, ante un estreptococo del grupo viridans o un enterococo resistente a vancomicina, debe sospecharse la posibilidad de que se trate de una infección por Leuconostoc'.

El tratamiento de elección en infecciones por Leuconostoc es la penicilina y la Ampicilina, aunque, se debe tener presente la posibilidad de mostrar una pobre respuesta clínica al tratamiento con antibioterapia perteneciente al grupo de los beta-lactámicos, a pesar de que en el antibiograma muestre sensibilidad a los mismos ${ }^{2,12}$. Otros antibióticos efectivos son la daptomicina, el linezolid o los carbapenemes, aunque, estos últimos pueden presentar un efecto insuficiente, tal como se describe en algunos casos en la literatura ${ }^{12}$. Alternativas a dicho tratamiento pueden ser el empleo de macrólidos, clindamicina, gentamicina o miociclina ${ }^{1}$.

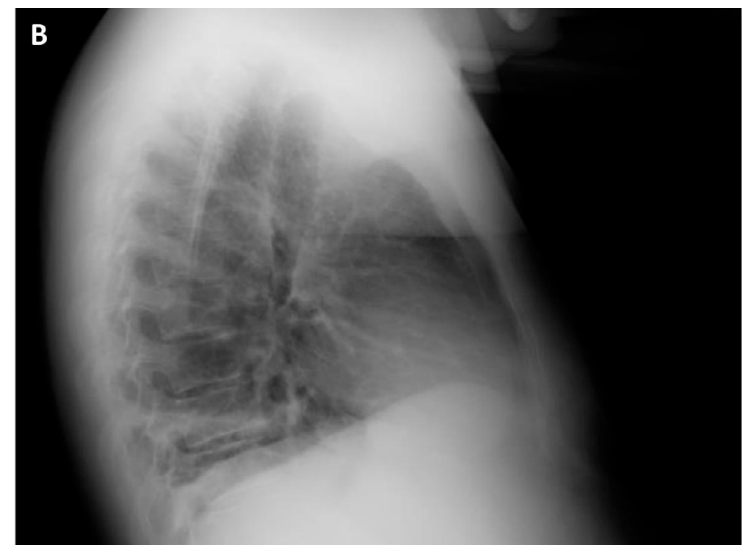

\section{CONCLUSIONES}

Las infecciones por Leuconostoc, especialmente la bacteriemia y la sepsis, son entidades poco conocidas y descritas, pero que afecta a pacientes inmunocomprometidos o con patología tumoral, cuyos cuadros clínicos pueden llegar a ser potencialmente graves. Por esta razón, es importante tenerlos en cuenta en el diagnóstico diferencial de sujetos que presentan dicha clínica y que asocian factores de riesgo para su desarrollo, sobre todo en aquellos casos en que se aíslen cocos gram-positivos sin respuesta clínica favorable a tratamiento con Vancomicina. No obstante, debe tenerse presente la posibilidad de afectar a pacientes inmunocompetentes.

\section{BIBLIOGRAFÍA}

1. Mensa J, Gatell JM, García-Sánchez JE, Letang E, López-Suñé E, Marco R. Guía de terapéutica antimicrobiana. Editorial Antares. 2019. Página 414.

2. 1. Lee M, Huang Y, Lee P, Liao C, Lai C, Lee L et al. Healthcare-associated bacteraemia caused by Leuconostoc species at a university hospital in Taiwan between 1995 and 2008. Journal of Hospital Infection. 2011; 78 (1): 45-49.

3. Buu-Hoi. Branger CA, Acar FJ. Vancomycin-resistant streptococci or Leuconostoc spp. Antimicrob Agents Chemother. 1985; 28: 458- 60.

4. Ino K, Nakase K, Suzuki K, Nakamura A, Fujieda A, N. Bacteremia due toLeuconostoc pseudomesenteroidesin a Patient with Acute Lymphoblastic Leukemia: Case Report and Review of the Literature. Case Reports in Hematology. 2016: 1-4.

5. Bou G, Saleta JL, Sáez JA, Tomás M, Valdezate S, Sousa D et al. Nosocomial Outbreaks caused by Leuconostoc mesenteroides subsp. Mesenteroides. Emerging Infectius Diseases. 2008. Vol 14 (6): 968-71.

6. Menegueti M, Gaspar G, Laus A, Basile-Filho A, Bellissimo-Rodrigues F, AuxiliadoraMartins M. Bacteremia by Leuconostoc mesenteroides in an immunocompetent patient with chronic Chagas disease: a case report. BMC Infectious Diseases. 2018; 18 (1).

7. Cuervo M S, Cortés L J, Rodríguez R E, Hormaza A N, Vargas S E. Leuconostoc spp en pacientes con cáncer: Estudio descriptivo. Revista chilena de infectología. 2008; 25 (3).

8. Ballesteros Sanz M, Ruiz De Alegría-Puig C, Fernández-Mazarrasa C, GutiérrezCuadra M. Bacteriemia y sepsis por Leuconostoc mesenteroides. Medicina Clínica. 2010; 134 (2): 87-88

9. Fauchais A, Roques S, Frébourg N, Druesne L, Verdonck A, Chassagne P et al. Leuconostoc, une cause rare d'infection opportuniste. La Revue de Médecine Interne. 2003; 24 (4): 268-69.

10. García-Granja P, López J, Ladrón R, Román J. Endocarditis infecciosa por Leuconostoc species. Revista Española de Cardiología. 2018; 71 (7): 592-594.

11. Holik H, Coha B, Sisko M, Tomic-paradzik M. Leuconostoc sp. Meningitis in a Patient Treated with Rituximab for Mantle Cell Lymphoma. Turkish Journal of Hematology. 2015; 32 (3): 271-74.

12. Ishiyama K, Yamazaki H, Nakao S, Ishiyama K, Senda Y, Yamauchi H. Leuconostoc bacteremia in three patients with malignancies. Journal of Infection and Chemotherapy. 2011; 17 (3): 412-18. 